\title{
GPS 控制网的优化设计及应用
}

刘春贵

上海汇谷岩土工程技术有限公司云南分公司

DOI:10.32629/gmsm.v2i4.210

[摘 要] 本文结合 GPS 控制网质量准则提出了 GPS 控制网优化设计的方法、步骤及其实践应用; 在限定精度、可靠性和费 用等质量标准下, 寻求网设计的最佳极值; 考虑质量合格的前提下,通过优化设计能使 GPS 控制测量达到减少工作量、提高经 济效益的目的。

[关键词] GPS 控制网；布设原则；优化设计；可靠性；精度

引言

近年 GPS 控制测量在各类大工程中已广泛应用, GPS 控 制网优化设计是控制测量基础性工作, 通常我们用三个准则 来评价网的质量、精度、可靠性和经济, 也即对控制网的设 计提出三个要求: (1)网元素的精度要求是可以在实际中达到 的; (2)可靠性尽可能高, 即一方面能探测出观测值中的粗差, 另一方面对未被发现的粗差有较强的抵抗能力; (3)点位的标 定和观测的实施满足一定的经济准则。由于 GPS 网的精度与 网的几何图形结构无关, 且与观测权相关甚小, 而影响精度 的主要因素是网中各点发出基线的数目及基线的权阵, 所以, 我们提出 GPS 控制网优化设计的概念。

\section{GPS 控制网的质量准则}

1.1 精度准则

GPS 控制网的高精度性是工程测量的基石，也是其最明 显的优势之一。在布设时, 要做到高精度性原则: 先确定 GPS 网的网形, 再根据 GPS 网的网形, 得到 GPS 网的设计矩阵 B, 从而得到 GPS 网的协因数阵 $Q=$ (BTPB), 由此做到 GPS 控制网 的高精度性原则。

\section{2 可靠性准则}

为了得到高的实现质量, 一个办法是对网进行第二次独 立观测 (复测), 在某些情况下, 这是需要和可行的。

1.3 灵敏度准则

灵敏度是一个相对概念, 即对于不同的变形向量具有不 同的下界值。一般将变形向量用表示其大小的模和表示其方 向的单位向量来表示, 即

$$
d=\|d\| \cdot g=a g
$$

1.4 费用准则

$$
\sum_{i=1}^{n} p_{i}=\min
$$

\section{GPS 控制网的优化设计措施}

2.1 控制网优化思想

2.1. 1 观测值的内部可靠性与观测值的精度、建网费用、
监测网的灵敏度和可区分性有密切的关系

2.1 .2 必须要有一定的多余观测, 多余观测数 $\mathrm{r}$ 愈大, 则 网的可靠性愈好, 但建网费用也愈高。

2.1 .3 在多余观测数一定的情况下, 观测值之间的精度 相差不要太大, 边角观测值之间的精度应基本匹配。

2.2 GPS 网的优化设计主要归结为两类内容的设计

2.2.1 GPS 网基准的优化设计

(1) 若网中点具有较准确的国家坐标系或地方坐标系 坐标, 可以通过它们所属坐标系与 WGS - 84 坐标系的转换 参数求得该点的 WGS -84 系坐标, 把它作为 GPS 网的固定位 置基准。

（2）若网中某点是 Doppler 点或 SLR 站, 由于其定位精度 较 GPS 伪距单点定位高得多, 可将其联至 GPS 网中作为一点 或多点基准。

(3) 若网中无任何其他类已知起算数据时, 可将网中一 点多次 GPS 观测的伪距坐标作为网的位置基准。

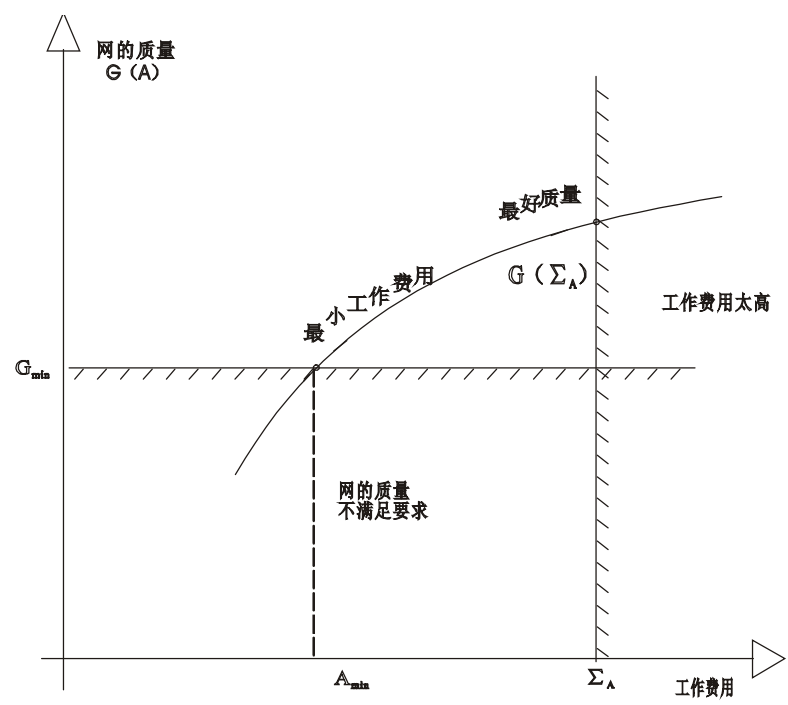

图 1 网的质量 $G(A)$ 为工作费用 $A$ 的函数

2.3 GPS 网图形结构强度的优化设计,包括: 网的精度 设计, 网的抗粗差能力的可靠性设计, 网发现系统差能力的 强度设计。 
3 GPS 控制网优化设计实例及步骤

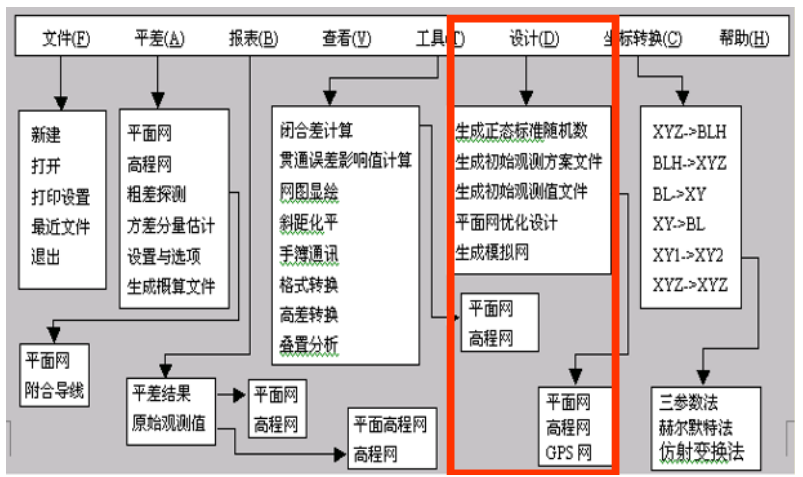

图 2 软件实施步骤

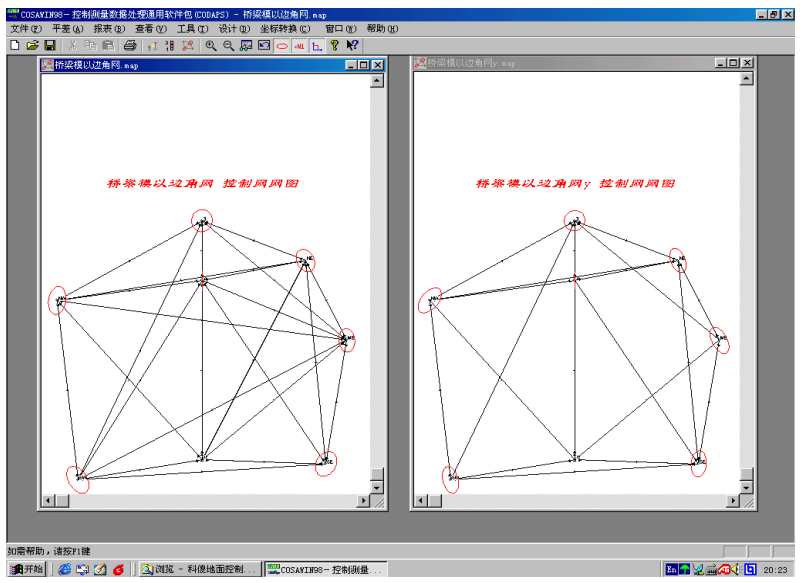

图 3 优化前后对比

本次以一座桥梁 GPS 控制网的优化过程为例, 网由 8 个 GPS 控制点组成的, 通过优化步骤介绍及优化前后对比, 使 GPS 控制网满足预期目的。

步骤如下:
(1) 确定观测精度和初始观测方案, 获取满足网形要求 的基准。

确定观测精度: 取仪器所能达到的最高精度。

初始观测方案: 所有可能观测的边和方向, “肥网” 或 “密网”。

(2) 模拟初始观测方案进行网形布置。

(3) 进行平差计算。

(4) 调整: 精度、可靠性乃至灵敏度计算结果进行分析, 首先确定观测精度的确定是否合理, 作适当调整。

\section{4 结论}

通过优化理论研究及实践应用, 在 GPS 控制网布网时, 考虑 GPS 网的布设原则及优化设计, 弄清楚 GPS 网基准化的 优化设计和图形结构强度的优化设计, GPS 控制网的布设和 优化设计就有了明确的方向和切实可行的措施。考虑质量合 格的前提下, 通过优化设计能使 GPS 控制测量达到减少工作 量、提高经济效益的目的。

\section{[参考文献]}

[1]李德仁.误差处理和可靠性理论:摄影测量平差的近 代发展 [M].测绘出版社,1988:6.

[2]赵长胜.GPS 控制网优化设计与数据处理 [J]. 现代测 绘,2003(:1):12-19.

[3]周秋生.测量控制网优化设计 [M]. 北京: 测绘出版 社,1992:63.

[4]刘基余.全球定位系统原理及其应用[M].测绘出版社, 1993:17.

[5]李征航.GPS 测量与数据处理 [M].武汉大学出版社,2013:3.

作者简介:

刘春贵(1984--), 男, 云南镇雄人, 汉族, 本科学历, 研究方 向：工程测量,从事工程测量工作。 